Sharif University of Technology
Scientia Iranica
Transactions E: Industrial Engineering
wCIENTIA

Research Note

\title{
Single-machine scheduling problem with convex multi-resource dependent processing times and job deadlines
}

\author{
M. Ziaee* \\ Department of Industrial Engineering, University of Bojnord, Bojnord, P.O. Box 94531-55111, Iran.
}

Received 31 May 2015; received in revised form 9 November 2015; accepted 26 January 2016

\section{KEYWORDS}

Scheduling;

Single-machine

scheduling problem;

Convex multi-resource

dependent processing

times;

Job deadlines;

Posynomial geometric

programming.

\begin{abstract}
In this paper, the single-machine scheduling problem with convex multiresource dependent processing times, subject to meeting job deadlines, is considered. The objective is to minimize the total cost, including the resource allocation costs and the fixed costs. We assume that the actual processing time of each job (task) is a function of the amount of resources allocated. Therefore, the decision variables of the model are: (1) resources allocated to the jobs; (2) total consumed resources; (3) processing times of the jobs; (4) start/completion times of the jobs. We reformulate and solve the problem using a posynomial geometric programming model. In the proposed exact solution method based on the geometric programming, the original problem of any size is reduced to a two-variable unconstrainted optimization problem, which can be easily solved by a simple grid search.
\end{abstract}

(C) 2017 Sharif University of Technology. All rights reserved.

\section{Introduction}

In many real scheduling problems, some parameters of the problem, such as the duration of tasks or their release times, can be controlled or improved by allocating more resources to them. Therefore, in these problems, two sub-problems should be solved sequentially or simultaneously: scheduling and resource allocation. In the resource allocation sub-problem, the actual processing time of tasks (jobs) is a function of the amount of resource allocated. This function usually has two forms: convex function and linear function (for other forms of the function see [1,2]). The linear form of the function for the single-machine scheduling problem has been studied by many papers in the literature [311]. However, the linear function does not obey the law of diminishing marginal product, in which productivity increases to the amount of resource at a decreasing

*. Tel.: +985832284611; Fax: +985832410700

E-mail address: ziaee@iust.ac.ir. rate [12]. Therefore, in this paper, the convex function is applied for the problem. This problem, i.e. the single-machine scheduling problem with convex resource dependent processing times, has been widely studied in the literature [13-21] due to the wide applicability of the convex resource consumption function and its fitness for many real problems [22]. However, almost all the existing studies on the problem consider only one resource allocation, and there are very few studies on multiple resources. Daniels [23] studied singlemachine scheduling problem with multiple resources available for processing times control. He considered a linear function for the resource dependent processing times and multi-objective function of minimizing the total amount of allocated resources and maximum job tardiness. Shabtay [24] studied the problem with a convex function and two resources available to control the processing times. The considered objective was the maximal lateness. Shabtay and Steiner [25] presented a survey of scheduling problems with controllable processing times. 
In this paper, we consider the single-machine scheduling problem with convex multi-resource dependent processing times, subject to meeting job deadlines. The objective is to minimize the total cost, including the resource allocation costs and the fixed costs. The remainder of this paper is organized as follows. A mathematical programming model is presented for the problem in Section 2. This mathematical model is then converted into a posynomial Geometric Programming (GP) model [26-28] in Section 3. An exact solution method is presented for the problem and the performance of the proposed method is evaluated by a numerical example in this section. Concluding remarks are given in the last section. follows:

The notations used throughout the paper are as

\section{Parameters and indices}

$n \quad$ Number of jobs,

$m \quad$ Number of resources,

$j \quad$ Index of jobs; $j=1, \cdots, n$,

$r \quad$ Index of resources; $r=1, \cdots, m$,

$p_{j}^{n} \quad$ Original (normal) processing time of job $j$,

$d_{j} \quad$ Deadline of job $j$,

fc Overall system fixed (overhead) cost per time unit,

$\mathrm{cr}_{r} \quad$ Unit cost of resource $r$ consumption,

$\operatorname{tar}_{r} \quad$ Total available resource $r$,

$R_{r j}^{n} \quad$ Normal (standard) consumption of resource $r$ for job $j$,

$w_{r j} \quad$ A constant positive parameter.

\section{Variables}

$C_{j} \quad$ Completion time of job $j$,

$P_{j}^{a} \quad$ Actual processing time of job $j$,

$\mathrm{TCR}_{r}$ Total amount of consumed resource $r$,

$R_{r j} \quad$ The amount of actual consumption of resource $r$ for job $j$.

The assumptions considered in this paper are as follows:

1. Jobs are independent of each other;

2. Setup and transportation times are negligible and job preemption is not allowed;

3. The machine (processor) is continuously available and can process at most one job at a time;

4. All the jobs have equal priorities;

5. All the jobs and the machine are available at time zero;

6. The processing time of job $j\left(P_{j}^{a}\right)$ is a function of resources, $R_{r j}, r=1,2, \cdots, m$, allocated to it. This function is as follows:

$$
P_{j}^{a}=p_{j}^{n} \cdot \prod_{r=1}^{m}\left[\left(\frac{R_{r j}^{n}}{R_{r j}}\right)^{w_{r j}}\right] \quad j=1,2, \cdots, n
$$

\section{Mathematical model}

The outputs or decision variables of the proposed mathematical model are:

1. Resources allocated to the jobs (tasks);

2. Total consumed resources;

3. Processing times of the jobs;

4. Start/completion times of the jobs.

Obviously, in order to meet the job deadlines, the sequence of the jobs should be based on EDD (Earliest Due Date) dispatching rule, in which the jobs are sequenced in non-decreasing order of their due dates [29]; and in scheduling sub-problem, only the start times of the jobs are determined. Therefore, we number the jobs in EDD order. The presented mathematical model for the problem is as follows:

$$
\begin{array}{ll}
\text { Minimize } \quad f c . C_{n}+\sum_{r=1}^{m}\left(c r_{r} . \mathrm{TCR}_{r}\right), \\
\sum_{j=1}^{n} R_{r j}=\mathrm{TCR}_{r} & r=1,2, \cdots, m, \\
\mathrm{TCR}_{r} \leq \operatorname{tar}_{r} & r=1,2, \cdots, m, \\
C_{1} \geq P_{1}^{a}, & j=2,3, \cdots, n, \\
C_{j} \geq P_{j}^{a}+C_{j-1} & j=1,2, \cdots, n, \\
C_{j} \leq d_{j} & \forall j, r . \\
C_{j}, P_{j}^{a}, R_{r j}, \mathrm{TCR}_{r} \geq 0
\end{array}
$$

The objective function is to minimize the total cost, including the resource allocation costs and the fixed costs. The first constraint set determines the total consumed resources. The second constraint set ensures that, for each resource, the total required resource does not exceed the total available resource. The third constraint guarantees that the start time of the first job is not less than zero. The fourth constraint set ensures that an operation does not overlap with both its predecessor and its successor operations. The fifth constraint set is used for meeting the deadlines. The last constraint set is for non-negativity limitations.

\section{Posynomial GP model}

We first rewrite $P_{j}^{a}$ in Eq. (1) as follows: 


$$
\begin{aligned}
P_{j}^{a} & =p_{j}^{n} \cdot \prod_{r=1}^{m}\left[\left(R_{r j}^{n}\right)^{w_{r j}}\right] \prod_{r=1}^{m}\left(R_{r j}^{-w_{r j}}\right) \\
& =\gamma_{j} \cdot \prod_{r=1}^{m}\left(R_{r j}^{-w_{r j}}\right) \\
j & =1,2, \cdots, n,
\end{aligned}
$$

such that:

$$
\gamma_{j}=p_{j}^{n} \cdot \prod_{r=1}^{m}\left[\left(R_{r j}^{n}\right)^{w_{r j}}\right], \quad j=1,2, \cdots, n .
$$

Model (2) is now reformulated as a posynomial GP model for the problem as follows:

$$
\begin{aligned}
& \text { Minimize } f c \cdot C_{n}+\sum_{r=1}^{m}\left(c r_{r} \cdot \mathrm{TCR}_{r}\right), \\
& \sum_{j=1}^{n}\left(R_{r j} \cdot \mathrm{TCR}_{r}^{-1}\right) \leq 1 \quad r=1,2, \cdots, m, \\
& \frac{1}{\operatorname{tar}_{r}} \cdot \mathrm{TCR}_{r} \leq 1 \quad r=1,2, \cdots, m, \\
& \gamma_{1} \cdot C_{1}^{-1} \cdot \prod_{r=1}^{m}\left(R_{r 1}^{-w_{r 1}}\right) \leq 1, \\
& C_{j-1} \cdot C_{j}^{-1}+\gamma_{j} \cdot C_{j}^{-1} \cdot \prod_{r=1}^{m}\left(R_{r j}^{-w_{r j}}\right) \leq 1 \\
& j=2,3, \cdots, n, \quad \forall j, r . \\
& \frac{1}{d_{j}} \cdot C_{j} \leq 1 \\
& C_{j}, R_{r j}, \mathrm{TCR}_{r}>0
\end{aligned}
$$

The degree of difficulty of a posynomial GP model is calculated as [(number of terms) - (number of variables $)-1]$. The degree of difficulty of model (5) is equal to $(m+2 n-1)$ [26-28], i.e. the problem is a very difficult problem. This means that the number of jobs $(n)$ has more effect on difficulty of the problem than the number of resources $(m)$. It should be noted that we applied variables $\mathrm{TCR}_{r}, \forall r$, in order to decrease the degree of difficulty of the model. The degree of difficulty of model (5) without variables $\mathrm{TCR}_{r}, \forall r$, is equal to $(m n+2 n-1)$. Similarly, given that in the third and fourth constraints of model $(2)$ the $(\geq)$ can be replaced with $(=)$, the variables $C_{j}$ can be eliminated from models (2) and (5). However, the degree of difficulty of this converted form of model (5) will increase to $2 m+\frac{n(n+1)}{2}-1$.

The dual formulation of model (5) is given below [26-28]:

$$
\begin{aligned}
\operatorname{Maximize} & \left(\frac{f c}{\alpha 1}\right)^{\alpha 1} \cdot \prod_{r=1}^{m}\left(\frac{c r_{r}}{\alpha 2_{r}}\right)^{\alpha 2_{r}} \cdot \prod_{r=1}^{m} \prod_{j=1}^{n}\left(\frac{\beta 3_{r}}{\alpha 3_{r j}}\right)^{\alpha 3_{r j}} \\
& \cdot \prod_{r=1}^{m}\left(\frac{1}{\operatorname{tar}_{r}}\right)^{\alpha 4_{r}} \cdot \prod_{j=2}^{n}\left(\frac{\beta 5_{j}}{\alpha 51_{j}}\right)^{\alpha 51_{j}} \\
& \prod_{j=1}^{n}\left(\frac{\gamma_{j} \cdot \beta 5_{j}}{\alpha 52_{j}}\right)^{\alpha 52_{j}} \cdot \prod_{j=1}^{n}\left(\frac{1}{d_{j}}\right)^{\alpha 6_{j}}
\end{aligned}
$$

subject to:

$$
\begin{aligned}
& \alpha 3_{r j}-w_{r j} . \alpha 52_{j}=0 \\
& j=1,2, \cdots,, \quad r=1,2, \cdots, m, \\
& -\alpha 52_{1}+\alpha 51_{2}+\alpha 6_{1}=0, \\
& -\alpha 51_{j}-\alpha 52_{j}+\alpha 51_{(j+1)}+\alpha 6_{j}=0 \\
& j=2, \cdots,(n-1), \\
& \alpha 1-\alpha 51_{n}-\alpha 52_{n}+\alpha 6_{n}=0, \\
& \alpha 2_{r}-\sum_{j=1}^{n}\left(\alpha 3_{r j}\right)+\alpha 4_{r}=0 \\
& \alpha 1+\sum_{r=1}^{m}\left(\alpha 2_{r}\right)=1, \quad r=1,2, \cdots, m, \\
& \sum_{j=1}^{n}\left(\alpha 3_{r j}\right)=\beta 3_{r} \quad r=1,2, \cdots, m, \\
& \alpha 51_{j}+\alpha 52_{j}=\beta 5_{j} \quad j=2,3, \cdots, n, \\
& \alpha 52_{1}=\beta 5_{1}, \alpha 2_{r}, \alpha 3_{r j}, \alpha 4_{r}, \alpha 51_{j}, \alpha 52_{j}, \alpha 6_{j}, \beta 3_{r}, \beta 5_{j}>0 .
\end{aligned}
$$

In the above model, all the constraints are linear. The dual variables $\alpha 1, \alpha 2_{r}, \alpha 3_{r j}, \alpha 4_{r}, \alpha 51_{j}, \alpha 52_{j}$, and $\alpha 6_{j}$ correspond to the terms of the primal problem (model (5)), respectively. Variables $\beta 3_{r}, \beta 5_{j}$ for $j=1, \beta 5_{j}$ for $j>1$ also correspond to the first, third, and fourth constraints of the primal problem, respectively.

Eqs. (7)-(9) yield the following equations, respectively:

$$
\begin{aligned}
\alpha 3_{r j} & =w_{r j} . \alpha 52_{j} \quad \forall j, r, \\
\alpha 52_{1} & =\alpha 51_{2}+\alpha 6_{1}, \\
\alpha 52_{j} & =-\alpha 51_{j}+\alpha 51_{(j+1)}+\alpha 6_{j}, \\
j & =2,3, \cdots,(n-1) .
\end{aligned}
$$


$\alpha 2_{r}$ can be computed from the following equation by using Eqs. (11) and (16)-(18):

$$
\begin{aligned}
\alpha 2_{r}= & \left\{w_{r 1} \cdot\left(\alpha 51_{2}+\alpha 6_{1}\right)+w_{r n} \cdot \alpha 52_{n}\right. \\
& \left.+\sum_{j=2}^{n-1}\left[w_{r j} \cdot\left(-\alpha 51_{j}+\alpha 51_{(j+1)}+\alpha 6_{j}\right)\right]\right\}-\alpha 4_{r} \\
r= & 1,2, \cdots, m .
\end{aligned}
$$

Similarly, we have the following equation by using Eqs. (12) and (17)-(19):

$$
\begin{aligned}
\alpha 1= & -\sum_{r=1}^{m}\left[\sum_{j=1}^{n}\left(w_{r j} \cdot \alpha 52_{j}\right)-\alpha 4_{r}\right] \\
= & 1-\sum_{r=1}^{m}\left\{w_{r 1} \cdot\left(\alpha 51_{2}+\alpha 6_{1}\right)+w_{r n} . \alpha 52_{n}\right. \\
& \left.+\sum_{j=2}^{n-1}\left[w_{r j} \cdot\left(-\alpha 51_{j}+\alpha 51_{(j+1)}+\alpha 6_{j}\right)\right]\right\} \\
& +\sum_{r=1}^{m}\left(\alpha 4_{r}\right) .
\end{aligned}
$$

The following equation is also obtained using Eqs. (13) and (16)-(18):

$$
\begin{aligned}
\beta 3_{r}= & \left\{w_{r 1} \cdot\left(\alpha 51_{2}+\alpha 6_{1}\right)+w_{r n} \cdot \alpha 52_{n}\right. \\
& \left.+\sum_{j=2}^{n-1}\left[w_{r j} \cdot\left(-\alpha 51_{j}+\alpha 51_{(j+1)}+\alpha 6_{j}\right)\right]\right\} \\
r= & 1,2, \cdots, m .
\end{aligned}
$$

Eqs. (14) and (15) yield:

$$
\beta 5_{j}= \begin{cases}\alpha 51_{(j+1)}+\alpha 6_{j} & j=2,3, \cdots,(n-1) \\ \alpha 51_{j}+\alpha 52_{j} & j=n\end{cases}
$$

and using Eqs. (10) and (20), we derive:

$$
\begin{aligned}
1 & -\sum_{r=1}^{m}\left\{w_{r 1} \cdot\left(\alpha 51_{2}+\alpha 6_{1}\right)+w_{r n} . \alpha 52_{n}\right. \\
& \left.+\sum_{j=2}^{n-1}\left[w_{r j} \cdot\left(-\alpha 51_{j}+\alpha 51_{(j+1)}+\alpha 6_{j}\right)\right]\right\} \\
& +\sum_{r=1}^{m}\left(\alpha 4_{r}\right)-\alpha 51_{n}-\alpha 52_{n}+\alpha 6_{n}=0 .
\end{aligned}
$$

The following single-constraint optimization problem now can be obtained by using the above equations:

$$
\begin{gathered}
\text { Maximize } \quad f\left(\alpha 4_{r}, \forall r ; \alpha 51_{j}, j=2,3, \cdots, n ;\right. \\
\left.\alpha 52_{n} ; \alpha 6_{j}, \forall j\right),
\end{gathered}
$$

subject to:

$$
\begin{aligned}
(23): & 1-\sum_{r=1}^{m}\left\{w_{r 1} \cdot\left(\alpha 51_{2}+\alpha 6_{1}\right)+w_{r n} . \alpha 52_{n}\right. \\
& \left.+\sum_{j=2}^{n-1}\left[w_{r j} \cdot\left(-\alpha 51_{j}+\alpha 51_{(j+1)}+\alpha 6_{j}\right)\right]\right\} \\
& +\sum_{r=1}^{m}\left(\alpha 4_{r}\right)-\alpha 51_{n}-\alpha 52_{n}+\alpha 6_{n}=0 .
\end{aligned}
$$

Problem (24) has $(2 n+m)$ variables, which are: $\alpha 4_{r}$, $\forall r ; \alpha 51_{j}, j=2,3, \cdots, n ; \alpha 52_{n} ; \alpha 6_{j}, \forall j$. The detailed objective function of this problem is given in Appendix 1. For simplicity, we now consider the following assumption:

$$
w_{r j}=w_{r} \quad \forall r, j .
$$

Theorem 1. In the optimal solution of problem (24), the following equation holds, assuming that $\alpha 51_{1}=0$ (note that $\alpha 51_{1}$ does not exist in the dual formulation of model (5)):

$$
\begin{aligned}
\sum_{r=1}^{m} & {\left[w_{r} \cdot \log \left(\frac{\alpha 52_{j}}{\alpha 52_{(j-1)}}\right)\right]+\log \left(\frac{\alpha 52_{j}}{\alpha 51_{j}}\right) } \\
& +\log \left(\frac{\alpha 51_{(j-1)}+\alpha 52_{(j-1)}}{\alpha 52_{(j-1)}}\right)+\log \left(\frac{\gamma_{(j-1)}}{\gamma_{j}}\right)=0 \\
j & =2,3, \cdots, n .
\end{aligned}
$$

Proof. According to the Karush-Kuhn-Tucker (KKT) conditions, Eq. (26) is true.

From Relation (26), the following equation can be easily obtained:

$$
\begin{aligned}
& \left(\frac{\alpha 52_{j}}{\alpha 52_{(j-1)}}\right)^{1+\sum_{r=1}^{m}\left(w_{r}\right)} \cdot \frac{\alpha 51_{(j-1)}+\alpha 52_{(j-1)}}{\alpha 51_{j}}=\frac{\gamma_{j}}{\gamma_{(j-1)}} \\
& j=2,3, \cdots, n .
\end{aligned}
$$

Theorem 2. In the optimal solution of problem (24), the following equation holds; cons is a constant:

$$
\frac{1}{\alpha 2_{r}}=\frac{f c}{e^{\text {cons }} \cdot c r_{r} \cdot \operatorname{tar}_{r}}+\frac{\sum_{r=1}^{m}\left(c r_{r} \cdot \operatorname{tar}_{r}\right)}{c r_{r} \cdot \operatorname{tar}_{r}} \quad \forall r .
$$


$\alpha 2_{r}$ is calculated by the following equation as mentioned earlier:

$$
\alpha 2_{r}=\sum_{j=1}^{n}\left(w_{r} . \alpha 52_{j}\right)-\alpha 4_{r} \quad \forall r .
$$

Proof. The Karush-Kuhn-Tucker conditions yield:

$$
\begin{aligned}
& \log \left(\frac{f c}{c r_{r} \cdot \operatorname{tar}_{r}}\right) \\
& \quad+\log \left[\frac{\sum_{j=1}^{n}\left(w_{r} \cdot \alpha 52_{j}\right)-\alpha 4_{r}}{1-\sum_{r=1}^{m} \sum_{j=1}^{n}\left(w_{r} \cdot \alpha 52_{j}\right)+\sum_{r=1}^{m}\left(\alpha 4_{r}\right)}\right]=\text { cons }
\end{aligned}
$$

$\forall r$.

Using Eqs. (29) and (30) yields:

$$
\log \left(\frac{\alpha 2_{r} \cdot f c}{\left[1-\sum_{r=1}^{m}\left(\alpha 2_{r}\right)\right] \cdot c r_{r} \cdot \operatorname{tar}_{r}}\right)=\mathrm{cons} \quad \forall r
$$

By summing Eq. (31) over $r$ and calculating the value of $\sum_{r=1}^{m}\left(f_{r}\right)$, the value of $f_{r}$ can be easily computed and Eq. (28) will be obtained.

Theorem 3. In the optimal solution of problem (24), the following equation holds, assuming that $\alpha 51_{1}=0$ :

$$
\begin{aligned}
& -\left\{-\log (f c)+\log \left[1-\sum_{r=1}^{m} \sum_{j=1}^{n}\left(w_{r} . \alpha 52_{j}\right)+\sum_{r=1}^{m}\left(\alpha 4_{r}\right)\right]\right. \\
& \left.+\log \left(\frac{\sum_{j=1}^{n} \alpha 52_{j}}{\alpha 52_{j}}\right)\right\}+\left\{\log \left(\frac{\gamma_{j}}{d_{j}}\right)\right. \\
& +\log \left(\frac{\alpha 51_{j}+\alpha 52_{j}}{\alpha 52_{j}}\right)+\sum_{r=1}^{m}\left[w_{r} \log \left(c r_{r}\right)\right] \\
& \left.-\sum_{r=1}^{m}\left[w_{r} \log \left(\sum_{j=1}^{n}\left(w_{r} . \alpha 52_{j}\right)-\alpha 4_{r}\right)\right]\right\} \\
& \left(\frac{-1}{\left(\sum_{r=1}^{m} w_{r}\right)}\right)=\text { cons } \\
& j=1,2, \cdots,(n-1) .
\end{aligned}
$$

Proof. According to the Karush-Kuhn-Tucker (KKT) conditions, Eq. (32) is true.

From Relation (32), the following equation can be easily obtained:

$$
\frac{\left(1-\sum_{r=1}^{m}\left(\alpha 2_{r}\right)\right)^{\sum_{r=1}^{m} w_{r}}}{\prod_{r=1}^{m}\left(\alpha 2_{r}^{w_{r}}\right)} \cdot \frac{\left(\sum_{j=1}^{n} \alpha 52_{j}\right)^{\sum_{r=1}^{m} w_{r}}}{\alpha 52_{j}^{\left(1+\sum_{r=1}^{m} w_{r}\right)}}
$$

$$
\begin{aligned}
& .\left(\alpha 51_{j}+\alpha 52_{j}\right)=\frac{d_{j}}{\gamma_{j} \cdot \prod_{r=1}^{m}\left(c r_{r}^{w_{r}}\right)} \cdot\left(\frac{f c}{e^{\mathrm{cons}}}\right)^{\sum_{r=1}^{m} w_{r}} \\
& j=1,2, \cdots,(n-1) .
\end{aligned}
$$

Theorem 4. In the optimal solution of problem (24), the following equation holds:

$$
\begin{aligned}
& -\left\{-\log (f c)+\log \left[1-\sum_{r=1}^{m} \sum_{j=1}^{n}\left(w_{r} . \alpha 52_{j}\right)+\sum_{r=1}^{m}\left(\alpha 4_{r}\right)\right]\right. \\
& \left.+\log \left(\frac{\sum_{j=1}^{n} \alpha 52_{j}}{\alpha 52_{n}}\right)\right\}+\left\{\log \left(\gamma_{n}\right)\right. \\
& +\log \left(\frac{\alpha 51_{n}+\alpha 52_{n}}{\alpha 52_{n}}\right)+\sum_{r=1}^{m}\left[w_{r} \log \left(c r_{r}\right)\right] \\
& \left.-\sum_{r=1}^{m}\left[w_{r} \log \left(\sum_{j=1}^{n}\left(w_{r} . \alpha 52_{j}\right)-\alpha 4_{r}\right)\right]\right\} \\
& \left(\frac{-1}{\left(\sum_{r=1}^{m} w_{r}\right)}\right)=\text { cons. }\left(\frac{1+\sum_{r=1}^{m} w_{r}}{\sum_{r=1}^{m} w_{r}}\right) \text {. }
\end{aligned}
$$

Proof. According to the Karush-Kuhn-Tucker (KKT) conditions, Eq. (34) is true.

From Relation (34), the following equation can be easily obtained.

$$
\begin{aligned}
\frac{\left(1-\sum_{r=1}^{m}(\alpha 2)\right)^{\sum_{r=1}^{m} w_{r}}}{\prod_{r=1}^{m}\left(\alpha 2_{r}^{w_{r}}\right)} \cdot \frac{\left(\sum_{j=1}^{n} \alpha 52_{j}\right)^{\sum_{r=1}^{m} w_{r}}}{\alpha 52_{n}^{\left(1+\sum_{r=1}^{m} w_{r}\right)}} \\
.\left(\alpha 51_{n}+\alpha 52_{n}\right)=\frac{\gamma_{n} \cdot\left[\prod_{r=1}^{m}\left(c r_{r}^{w_{r}}\right)\right] \cdot\left(e^{\sum_{r=1}^{m} w_{r}}\right)^{1+\sum_{r=1}^{m} w_{r}}}{} .
\end{aligned}
$$

\subsection{Some useful relations}

i. Using Eqs. (28) and (33), we have the following relation between $\alpha 51_{j}$ and $\alpha 52_{j}(2 \leq j \leq(n-1))$, assuming that $\alpha 51_{1}=0$ (Note that $\alpha 51_{1}$ does not exist in the dual formulation of Model (5).): 


$$
\begin{gathered}
\alpha 51_{j}=\frac{d_{j}}{\gamma_{j}} \cdot \prod_{r=1}^{m}\left(\operatorname{tar}_{r}^{w_{r}}\right) \cdot \frac{\alpha 52_{j}^{\left(1+\sum_{r=1}^{m} w_{r}\right)}}{\left(\sum_{j=1}^{n} \alpha 52_{j}\right)^{\sum_{r=1}^{m} w_{r}}}-\alpha 52_{j} \\
j=1,2, \cdots,(n-1) .
\end{gathered}
$$

ii. Using Relations (27) and (36), the following equation is obtained for $\alpha 52_{j}, j=2,3, \cdots,(n-1)$.

$$
\begin{aligned}
& \frac{\alpha 52_{j}}{\sum_{j=1}^{n} \alpha 52_{j}}=\left[\frac{\gamma_{j}}{\left(d_{j}-d_{(j-1)}\right) \cdot \prod_{r=1}^{m}\left(\operatorname{tar}_{r}^{w_{r}}\right)}\right]^{\frac{1}{\sum_{n=1}^{m} w_{r}}} \\
& \quad j=2,3, \cdots,(n-1) .
\end{aligned}
$$

iii. Using Relations (36) and (37), we have the following relation between $\alpha 51_{j}$ and $\alpha 52_{j}(2 \leq j \leq$ $(n-1))$ :

$$
\alpha 51_{j}=\alpha 52_{j} \cdot \frac{d_{(j-1)}}{\left(d_{j}-d_{(j-1)}\right)} \quad j=2,3, \ldots,(n-1) .
$$

iv. Using Eqs. (27) and (38), we have the following relation between $\alpha 52_{j}$ and $\alpha 52_{(j-1)}(2 \leq j \leq(n-$ $1)$ ), assuming that $d_{0}=0$ :

$$
\begin{aligned}
& \frac{\alpha 52_{j}}{\alpha 52_{(j-1)}}=\left(\frac{\gamma_{j}}{\gamma_{(j-1)}} \cdot \frac{d_{(j-1)}-d_{(j-2)}}{d_{j}-d_{(j-1)}}\right)^{\frac{1}{\sum_{n}\left(w_{r}\right)}} \\
& j=2,3, \cdots,(n-1) .
\end{aligned}
$$

v. Using Relations (28) and (35), we have the following relation between $\alpha 51_{n}$ and $\alpha 52_{n}$ :

$$
\begin{aligned}
\alpha 51_{n}= & \frac{1}{e^{\text {cons }} \cdot \gamma_{n}} \cdot \prod_{r=1}^{m}\left(\operatorname{tar}_{r}^{w_{r}}\right) \\
& \cdot \frac{\alpha 52_{n}\left(1+\sum_{r=1}^{m} w_{r}\right)}{\left(\sum_{j=1}^{n} \alpha 52_{j}\right)^{\sum_{r=1}^{m} w_{r}}-\alpha 52_{n} .}
\end{aligned}
$$

vi. Using Relations (36) and (37), the following equation is obtained for $\alpha 52_{n}$ :

$$
\begin{aligned}
\alpha 52_{n}= & \left(\sum_{j=1}^{n} \alpha 52_{j}\right)\left[1-\left(\left(\prod_{r=1}^{m}\left(\operatorname{tar}_{r}^{w_{r}}\right)\right)^{\frac{-1}{\sum_{r=1}^{m} w_{r}}}\right)\right. \\
& \cdot\left(\left(\frac{d_{1}}{\gamma_{1}}\right)^{\frac{-1}{\sum_{r=1}^{m} w_{r}}}\right. \\
& \left.\left.+\sum_{j=2}^{n-1}\left(\left(\frac{\gamma_{j}}{d_{j}-d_{j-1}}\right)^{\frac{1}{\sum_{r=1}^{m} w_{r}}}\right)\right)\right] .
\end{aligned}
$$

vii. Using Eqs. (8)-(10), $\alpha 1$ can be obtained from the following equation.

$$
\alpha 1=\left[\sum_{j=1}^{n}\left(\alpha 52_{j}\right)\right]-\left[\sum_{j=1}^{n}\left(\alpha 6_{j}\right)\right] .
$$

\subsection{Solution method}

Using Eqs. (28) and (29), $\alpha 4_{r}, \forall r$, can be written as a function of $\sum_{j=1}^{n}\left(\alpha 52_{j}\right)$ and cons, as follows:

$$
\begin{aligned}
\alpha 4_{r}= & {\left[\sum_{j=1}^{n}\left(\alpha 52_{j}\right)\right] . w_{r} } \\
& -\frac{1}{\frac{f c}{e^{c o n s} \cdot c r_{r} \cdot \operatorname{tar}_{r}}+\frac{\sum_{r=1}^{m}\left(c r_{r} \cdot \operatorname{tar}_{r}\right)}{c r_{r} \cdot \operatorname{tar}_{r}}} \forall r .
\end{aligned}
$$

Also, $\alpha 51_{j}, j=2,3, \cdots,(n-1)$, can be written as a function of $\sum_{j=1}^{n}\left(\alpha 52_{j}\right)$ using Eqs. (37) and (38):

$$
\begin{aligned}
\alpha 51_{j}= & \sum_{j=1}^{n}\left(\alpha 52_{j}\right) \cdot\left[\frac{\gamma_{j}}{\left(d_{j}-d_{(j-1)}\right) \cdot \prod_{r=1}^{m}\left(\operatorname{tar}_{r}^{w_{r}}\right)}\right]^{\frac{1}{\sum_{r=1}^{m} w_{r}}} \\
& \cdot \frac{d_{(j-1)}}{\left(d_{j}-d_{(j-1)}\right)} \quad j=2,3, \cdots,(n-1) . \quad(44)
\end{aligned}
$$

Using Eqs. (9), (37), and (38), $\alpha 6_{j}, j=1,2, \cdots,(n-1)$, can be written as a function of $\sum_{j=1}^{n}\left(\alpha 52_{j}\right)$, assuming that $d_{0}=0$ :

$$
\begin{aligned}
& \alpha 6_{j}=\frac{\left[\sum_{j=1}^{n}\left(\alpha 52_{j}\right)\right] \cdot d_{j}}{\left[\prod_{r=1}^{m}\left(\operatorname{tar}_{r}^{w_{r}}\right)\right]_{r=1}^{\frac{1}{m} w_{r}}} \cdot\left\{\left(\frac{\frac{\gamma_{j}^{\sum_{j=1}^{m} w_{r}}}{\left(d_{j}-d_{(j-1)}\right){ }^{1+\frac{1}{\sum_{r=1}^{m} w_{r}}}}}{\lambda}\right)\right. \\
& \left.-\left(\frac{\frac{1}{\gamma_{j+1}^{\substack{m \\
r=1}}}}{\left(d_{j+1}-d_{j}\right)^{1+\frac{1}{\sum_{r=1}^{m} w_{r}}}}\right)\right\} \\
& j=1,2, \cdots,(n-1) .
\end{aligned}
$$

Also, $\alpha 52_{n}$ can be written as a function of $\sum_{j=1}^{n}\left(\alpha 52_{j}\right)$ and $\alpha 51_{n}$ as a function of $\sum_{j=1}^{n}\left(\alpha 52_{j}\right)$ and cons, using Eqs. (40) and (41).

From Eqs. (12) and (28), we deduce:

$$
\begin{aligned}
\alpha 1 & =1-\sum_{r=1}^{m}\left(\alpha 2_{r}\right) \\
& =1-\sum_{r=1}^{m}\left(\frac{e^{\text {cons }} \cdot c r_{r} \cdot \operatorname{tar}_{r}}{f c+e^{\text {cons }} \cdot \sum_{r=1}^{m}\left(c r_{r} \cdot \operatorname{tar}_{r}\right)}\right) .
\end{aligned}
$$


And using Eqs. (10), (41), and (46), $\alpha 6_{n}$ can be written as a function of $\sum_{j=1}^{n}\left(\alpha 52_{j}\right)$, as follows:

$$
\alpha 6_{n}=\alpha 51_{n}+\alpha 52_{n}-\alpha 1 .
$$

Therefore, using Eqs. (40)-(43) and (47), problem (24) with $(2 n+m)$ variables is finally reduced to the following single-constraint optimization problem with only two variables: $\sum_{j=1}^{n}\left(\alpha 52_{j}\right)$ and cons:

$$
\text { Maximize } f^{\prime}\left(\sum_{j=1}^{n}\left(\alpha 52_{j}\right), \text { cons }\right) \text {. }
$$

Subject to:

$$
g\left(\sum_{j=1}^{n}\left(\alpha 52_{j}\right), \text { cons }\right)=0 .
$$

Problem (52) can be solved by applying the Lagrangian relaxation technique as follows:

$$
\begin{aligned}
\text { Maximize } & f^{\prime}\left(\sum_{j=1}^{n}\left(\alpha 52_{j}\right), \text { cons }\right) \\
& -\lambda . g\left(\sum_{j=1}^{n}\left(\alpha 52_{j}\right), \text { cons }\right),
\end{aligned}
$$

where $\lambda$ is the Lagrangian multiplier. It can be shown that cons and $\lambda$ are the same. Therefore, problem (49) has only two variables, $\sum_{j=1}^{n}\left(\alpha 52_{j}\right)$ and $\lambda$.

The problem is finally converted to the following two-variable unconstrained optimization problem for any size of the original problem, even for very large sizes of the original one $\left(X=\sum_{j=1}^{n}\left(\alpha 52_{j}\right), Y=\lambda\right)$.

$$
\begin{aligned}
\text { Maximize } & a_{1} \cdot X+a_{2} \cdot X \cdot \ln (X)+a_{3} \cdot X \cdot e^{-Y} \\
& -a_{4} \cdot X \cdot\left(a_{5} \cdot e^{-Y}-1\right) \cdot \ln \left(1-a_{6} \cdot e^{Y}\right) \\
& -a_{7} \cdot X \cdot Y+\frac{1}{1+a_{8} \cdot e^{-Y}} \cdot\left(a_{9}-Y\right) \\
& +\ln \left(1+a_{10} \cdot e^{Y}\right)+a_{11} .
\end{aligned}
$$

Also, we can obtain some lower and upper limits for $X$ and $Y$, for example:

$$
1-a_{6} \cdot e^{Y}>0 \rightarrow Y<-\ln \left(a_{6}\right)
$$

that lead to decrease in the solving time of the problem.

Optimal solution of this relaxed dual problem is applied to obtain the original decision variables corresponding to the primal problem (5). Suppose that $\left[\sum_{j=1}^{n}\left(\alpha 52_{j}\right)\right]^{*}$ and $\lambda^{*}$ (or cons s $^{*}$ ) are the optimal values of variables of problem (48), and let $f^{*}$ be the optimal value of its objective function. The optimal values of all dual variables corresponding to problem (24), i.e. $\alpha 4_{r}^{*}$, $\forall r ; \alpha 51_{j}^{*}, j=2,3, \cdots, n ; \alpha 52_{n}^{*} ; \alpha 6_{j}^{*}, \forall j$, can be easily obtained by Eqs. (40)-(43) and (47). Also, the optimal values of all remaining dual variables corresponding to the original dual problem can be computed by Eqs. (16) to (22). Now, the optimal primal variables can be computed by the relationships between the primal and dual problems of GP as follows:

$$
\begin{aligned}
& C_{n}^{*}=\frac{\alpha 1^{*} \cdot f^{*}}{f c} \quad \text { if } \quad \alpha 1^{*}>0 \\
& \mathrm{TCR}_{r}^{*}=\frac{\alpha 2_{r}^{*} \cdot f^{*}}{c r_{r}} \quad \forall r \quad \text { if } \quad \alpha 2_{r}^{*}>0, \\
& R_{r j}^{*}=\mathrm{TCR}_{r}^{*} \cdot \frac{\alpha 3_{r j}^{*}}{\beta 3_{r}^{*}}=\frac{\alpha 2_{r}^{*} \cdot f^{*}}{c r_{r}} \cdot \frac{\alpha 3_{r j}^{*}}{\beta 3_{r}^{*}} \quad \forall r, j \\
& \text { if } \alpha 2_{r}^{*}, \alpha 3_{r j}^{*}>0, \\
& \mathrm{TCR}_{r}^{*}=\operatorname{tar}_{r} \quad \forall r \quad \text { if } \quad \alpha 4_{r}^{*}>0, \\
& \frac{C_{j-1}^{*}}{C_{j}^{*}}=\frac{\alpha 51_{j}^{*}}{\beta 5_{j}^{*}} \quad j=2,3, \cdots, n \quad \text { if } \quad \alpha 51_{j}^{*}>0, \\
& C_{j}^{*}=\frac{\gamma_{j} \cdot \prod_{r=1}^{m}\left(R_{r j}^{*^{-} w r}\right) \cdot \beta 5_{j}^{*}}{\alpha 52_{j}^{*}} \quad \forall j \quad \text { if } \quad \alpha 52_{j}^{*}>0, \\
& C_{j}^{*}=d_{j} \quad \forall j \quad \text { if } \quad \alpha 6_{j}^{*}>0 .
\end{aligned}
$$

In the next section, we use the proposed method to solve a numerical example.

\subsection{Numerical example}

The parameter values for the numerical example are as follows:

$$
\begin{aligned}
& n=5, \quad m=2 ; \quad d_{1}=1, \quad d_{2}=5 \\
& d_{3}=14, \quad d_{4}=25, \quad d_{5}=60 ; \quad \gamma_{1}=30000 \\
& \gamma_{2}=60000, \quad \gamma_{3}=40000, \quad \gamma_{4}=70000, \quad \gamma_{5}=10000 \\
& w_{1}=1.2, \quad w_{2}=1.1 ; \quad \operatorname{tar}_{1}=220, \quad \operatorname{tar}_{2}=290 \\
& c r_{1}=60, \quad c r_{2}=35 ; \quad f c=600 .
\end{aligned}
$$

This example is solved by using the procedure explained in this section. The procedure first solves twovariable unconstrainted optimization problem (53). The results are as follows:

$$
\begin{aligned}
& {\left[\sum_{j=1}^{n}\left(\alpha 52_{j}\right)\right]^{*}=5.1224962, \quad \lambda^{*}=-3.904,} \\
& f^{\prime *}=53114.8
\end{aligned}
$$


Using Eqs. (45)-(49) and (51), the optimal values of all dual variables corresponding to Problem (24) can be obtained as follows:

$$
\begin{array}{ll}
\alpha 4_{1}^{*}=5.898474, & \alpha 4_{2}^{*}=5.443648 ; \\
\alpha 51_{2}^{*}=0.333665, & \alpha 51_{3}^{*}=0.4369322, \\
\alpha 51_{4}^{*}=1.170035, & \alpha 51_{5}^{*}=0.2824099 ; \\
\alpha 52_{5}^{*}=0.2779718 ; & \\
\alpha 6_{1}^{*}=1.470406, & \alpha 6_{2}^{*}=1.231394, \\
\alpha 6_{3}^{*}=0.0534, & \alpha 6_{4}^{*}=1.806939, \\
\alpha 6_{5}^{*}=0 . &
\end{array}
$$

And the optimal values of all remaining dual variables corresponding to the original dual problem can be computed by Eqs. (16) to (22) as follows,

$$
\begin{array}{ll}
\alpha 1_{1}^{*}=0.5603817 ; & \\
\alpha 2_{1}^{*}=0.2485209, & \alpha 2_{2}^{*}=0.1910975 ; \\
\alpha 3_{1,1}^{*}=2.164885, & \alpha 3_{1,2}^{*}=1.601593, \\
\alpha 3_{1,3}^{*}=0.9437739, & \alpha 3_{1,4}^{*}=1.103177, \\
\alpha 3_{1,5}^{*}=0.3335661 ; & \\
\alpha 3_{2,1}^{*}=1.984478, & \alpha 3_{2,2}^{*}=1.468127, \\
\alpha 3_{2,3}^{*}=0.8651261, & \alpha 3_{2,4}^{*}=1.011246, \\
\alpha 3_{2,5}^{*}=0.3057689 ; & \\
\alpha 52_{1}^{*}=1.804071, & \alpha 52_{2}^{*}=1.334661, \\
\alpha 52_{3}^{*}=0.7864783, & \alpha 52_{4}^{*}=0.9193141 ; \\
\beta 3_{1}^{*}=6.146996, & \beta 3_{2}^{*}=5.634746 ; \\
\beta 5_{1}^{*}=1.804071, & \beta 5_{2}^{*}=1.668326, \\
\beta 5_{3}^{*}=1.223411, & \beta 5_{4}^{*}=2.089349, \\
\beta 5_{5}^{*}=0.5603812 . &
\end{array}
$$

Now, the optimal primal variables can be computed by Eqs. (54) to (60), as follows:

$$
\begin{aligned}
& C_{1}^{*}=1, \quad C_{2}^{*}=5, \quad C_{3}^{*}=14, \\
& C_{4}^{*}=25, \quad C_{5}^{*}=49.607 ; \\
& R_{1,1}^{*}=77.48095, \quad R_{1,2}^{*}=57.32081, \\
& R_{1,3}^{*}=33.77755, \quad R_{1,4}^{*}=39.48257, \\
& R_{1,5}^{*}=11.93811, \quad R_{2,1}^{*}=102.1340,
\end{aligned}
$$

$$
\begin{array}{ll}
R_{2,2}^{*}=75.55926, & R_{2,3}^{*}=44.52495, \\
R_{2,4}^{*}=52.04521, & R_{2,5}^{*}=15.73660 ; \\
\mathrm{TCR}_{1}^{*}=220, & \mathrm{TCR}_{2}^{*}=290 .
\end{array}
$$

\section{Conclusion}

In many task scheduling problems in the real world, processing of tasks (jobs) requires some continuously divisible constrained nonrenewable resources, such as manpower, energy, fuel, gas, oxygen, catalyst, raw materials, money, etc. In theses situations, a good resource allocation may considerably improve the system performance and decrease the total cost. In this paper, the single-machine scheduling problem with resource dependent processing times, subject to meeting job deadlines, was studied. We considered multi-resource allocation problem that was a more general and much more complex problem, instead of single-resource allocation problem extensively studied in the literature. This multi-resource problem is very flexible to apply in the real problems and can be used for even noncommon limited resources. The problem was more generalized by assuming that the job processing times were a convex function of the amounts of resources allocated. This form of the function is more applicable in the real problems in comparison with the linear form, widely studied in the literature. The objective was to minimize the total cost, including the resource allocation costs and the fixed costs. We presented a solution method based on geometric programming for the problem. In the proposed exact method, the original problem of any size was reduced to a twovariable unconstrainted optimization problem, which could easily be solved by a simple grid search. The proposed method can be applied for many real-world resource allocation problems with task deadlines.

\section{References}

1. Chen, Z.-L., Lu, Q. and Tang, G. "Single machine scheduling with discretely controllable processing times", Operations Research Letters, 21, pp. 69-76 (1997).

2. Grigoriev, A., Sviridenko, M. and Uetz, M. "Machine scheduling with resource dependent processing times", Mathematical Programming, 110(1), pp. 209228 (2007).

3. Cheng, T.C.E. and Kovalyov, M.Y. "Single machine batch scheduling with deadlines and resource dependent processing times", Operations Research Letters, 17, pp. 243-249 (1995).

4. Janiak, A. and Kovalyov, M.Y. "Single machine scheduling subject to deadlines and resource dependent processing times", European Journal of Operational Research, 94, pp. 284-291 (1996). 
5. Janiak, A., Kovalyov, M.Y. and Portmann, M.-C. "Single machine group scheduling with resource dependent setup and processing times", European Journal of Operational Research, 162, pp. 112-121 (2005).

6. Choi, B.-C., Yoon, S.-H. and Chung, S.-J. "Single machine scheduling problem with controllable processing times and resource dependent release times", European Journal of Operational Research, 181, pp. 645-653 (2007).

7. Wang, J.-B. and Xia, Z.-Q. "Single machine scheduling problems with controllable processing times and total absolute differences penalties", European Journal of Operational Research, 177, pp. 638-645 (2007).

8. Wang, J.-B., Wang, M.-Z. and Ji, P. "Scheduling jobs with processing times dependent on position, starting time, and allocated resource", Asia-Pacific Journal of Operational Research, 29(5), 1250030 (15 pages) (2012).

9. Wei, C.-M., Wang, J.-B. and Ji, P. "Single-machine scheduling with time-and-resource-dependent processing times", Applied Mathematical Modelling, 36, pp. 792-798 (2012).

10. Kayvanfar, V., Mahdavi, I. and Komaki, G.M. "Single machine scheduling with controllable processing times to minimize total tardiness and earliness", Computers \& Industrial Engineering, 65, pp. 166-175 (2013).

11. Mor, B. and Mosheiov, G. "Batch scheduling of identical jobs with controllable processing times", Computers \& Operations Research, 41, pp. 115-124 (2014).

12. Maurice, S.C. and Phillips, O.R., Economic Analysis: Theory and Application, IRWIN, Homewood (1986).

13. Shabtay, D. and Kaspi, M. "Minimizing the total weighted flow time in a single machine with controllable processing times", Computers \& Operations Research, 31, pp. 2279-2289 (2004).

14. Kaspi, M. and Shabtay, D. "A bicriterion approach to time/cost trade-offs in scheduling with convex resource-dependent job processing times and release dates", Computers \& Operations Research, 33, pp. 3015-3033 (2006).

15. Shabtay, D. and Steiner, G. "The single-machine earliness-tardiness scheduling problem with due date assignment and resource-dependent processing times", Annals of Operations Research, 159, pp. 25-40 (2008).

16. Wang, D., Wang, M.-Z. and Wang, J.-B. "Singlemachine scheduling with learning effect and resourcedependent processing times", Computers \& Industrial Engineering, 59, pp. 458-462 (2010).

17. Koulamas, C., Gupta, S. and Kyparisis, G.J. "A unified analysis for the single-machine scheduling problem with controllable and non-controllable variable job processing times", European Journal of Operational Research, 205, pp. 479-482 (2010).

18. Hsu, C.-J. "Due-date assignment and single-machine resource allocation scheduling with deteriorating jobs", IEEE 18Th International Conference on Industrial Engineering and Engineering Management - IE\&EM 2011, Part 2, pp. 787-791 (2011).
19. Zhu, Z., Sun, L., Chu, F. and Liu, M. "Single-machine group scheduling with resource allocation and learning effect", Computers \& Industrial Engineering, 60, pp. 148-157 (2011).

20. Wang, X.-R. and Wang, J.-J. "Single-machine scheduling with convex resource dependent processing times and deteriorating jobs", Applied Mathematical Modelling, 37, pp. 2388-2393 (2013).

21. Yin, Y., Cheng, T.C.E., Cheng, S.-R. and Wu, C.C. "Single-machine batch delivery scheduling with an assignable common due date and controllable processing times", Computers \& Industrial Engineering, 65, pp. 652-662 (2013).

22. Monma, C.L., Schrijver, A., Todd, M.J. and Wei, V.K. "Convex resource allocation problems on directed acyclic graphs: duality, complexity, special cases and extensions", Mathematics of Operations Research, 15, pp. 736-748 (1990).

23. Daniels, R.L. "A multi-objective approach to resource allocation in single machine scheduling", European Journal of Operational Research, 48, pp. 226-241 (1990).

24. Shabtay, D. "Single and two-resource allocation algorithms for minimizing the maximal lateness in a single machine", Computers \& Operations Research, 31, pp. 1303-1315 (2004).

25. Shabtay, D. and Steiner, G. "A survey of scheduling with controllable processing times", Discrete Applied Mathematics, 155, pp. 1643-1666 (2007).

26. Duffin, R.J., Peterson, E.L. and Zener, C., Geometric Programming-Theory and Application, New York, John Wiley \& Sons (1967).

27. Rardin, R.L., Optimization in Operations Research, New Jersey, Prentice-Hall (1998).

28. Boyd, S., Kim, S.-J., Vandenberghe, L. and Hassibi, A. "A tutorial on geometric programming", Optimization and Engineering, 8, pp. 67-127 (2007).

29. Pinedo, M., Scheduling: Theory, Algorithms and Systems, Englewood Cliffs, New Jersey: Prentice-Hall (2002).

\section{Biography}

Mohsen Ziaee accomplished his MSc in Industrial Engineering at University of Tehran, Tehran, Iran (1999-2001), and PhD in Industrial Engineering at Iran University of Science and Technology, Tehran, Iran (2003-2008). Currently, he is an Assistant Professor at Industrial Engineering Department, University of Bojnord, Bojnord, Iran. His research interests are production planning and scheduling, multi-criteria decision making, applied operations research, simulation, and artificial intelligence techniques in the areas of manufacturing systems design. 